\title{
Technology for Extracting Effective Components from
}

\section{Fish Scale}

\author{
Wu Suo-Lian ${ }^{1}$, Kang Huai-Bin ${ }^{2}$ and Li Dong-Jiao ${ }^{1}$ \\ 1. School of Medical, Ezhou Polytechnic, Ezhou 436000, China \\ 2. School of Food Engineering and Biotechnology, Henan University of Science and Technology, Luoyang 471000, China
}

\begin{abstract}
The research of the category and extraction methods of effective ingredients of fish scale was reviewed at home and abroad. The extraction process of fish scale was studied, including collagen, hydroxyapatite, lecithin, guanine etc. The different extraction methods and the components were analyzed. The research of effective ingredients of fish scales was reviewed as theory basis to further research and comprehensive development.
\end{abstract}

Key words: Fish scale, effective components, collagen, hydroxyapatite, lecithin, guanine.

\section{Introduction}

The fishery output has been ranked first in succession in the world. In recent years, the annual output of aquatic products has been around 67 million tons. The spent material would be above $30 \%$ of total amount of aquatic products, the fish scale is about $5 \%$ of the spent material [1]. Most of the spent material can be used as a feedstuff or be discarded directly as waste, not only is the environment polluted but also a great deal of resources are wasted. To making full use of the value ingredient of the spent material, it is necessary to study the extraction of effective components from fish scales to improve economic benefit of low additional value fish.

Fish scales contain a mass of organic/inorganic components. Using the modern technology of biochemistry, the comprehensive utilization of fish scales was researched. These kinds of highly valuable biochemical product were extracted, including collagen, hydroxyapatite, lecithin, guanine etc. [2].

Corresponding author: Wu Suo-Lian, master's degree, research field: food processing.

Fund projects: 1 . Supported by Scientific Research Project in School-level of Ezhou Polytechnic, Grant No. 2016YBA51;

2. Supported by Science and Technology Research Project of Hubei Provincial Department of Education, Grant No. B2017531.
The biochemical products will be wildly used in medicine, food and cosmetic industry and so on. The effective ingredients from fish scales have attracted scholars' attention [3]. In recent reports, the extraction process of effective ingredients was paid more attention on the single extraction than the comprehensive extraction which is an important research direction. The research of effective ingredients of fish scales was reviewed as theory basis to further research and comprehensive development.

\section{The Composition and Structure of the Fish Scale}

The fish scale and bone have a similar structure, the fish collagen dermis has gradually evolved into the derivatives bone [4]. There are plenty of organic /inorganic components in the fish scale. The organic components were about $40 \%-55 \%$, including collagen, scleroprotein, lecithin, fat and variety of vitamins, etc.; the percentage of organic components was about 7\%-25\%, including hydroxyapatite and calcium phosphate. Toshiyuki Ikoma et al. [5] researched the microstructure of fish scales from Pafrus major by transmission electron microscope, scanning electron microscope, X-ray in 2003. The fish scale of Pafrus major was comprised of the I type collagen with 
three-screws structure and the hydroxyapatite. Results of orthogonal action indicated that fish scale is composed of inner fibrillary plate and outer hydroxylapatite layer, the fiber of collagen was adhered by apatite. Deashing and decalcification must be done in order to remove the organic impurities and metal elements before fish collagen was extracted. High quality collagen can be obtained.

\section{Research the Progress of the Effective Composition of Fish Scales}

\subsection{Extraction of Collagen from Fish Scale}

Collagen is a kind of structural protein of the extracellular matrix which can be used as a bonding material. According to the biological properties of collagen, it can be isolated by the chemical degradation or enzyme degradation. The extraction process: the clean fish scales were soaked in $10 \%$ $\mathrm{NaCl}$ solution which could remove the other protein. The fish scale was extracted by different kinds of maceration extract after it had been decalcified by acid method or EDTA method. We could obtain different collagen with different molecular weight ranges. It was salt out at the pI and dissolution again. The pure collagen was prepared after being dialyzed and lyophilized. The extraction of gelatin was done by heating by which the I type collagen with three-screws structure was opened. The pure gelatin was obtained after spray drying the condensed extraction of liquid.

\subsubsection{Decalcification of the Fish Scale}

The methods of decalcification include acid-decalcification, EDTA-decalcification and other methods decalcification of physical auxiliary from fish scales (such as ultrasonic and microwave). Inclusion of hydrochloric acid, citric acid and lactic acid, was usually used in the acid-decalcification process. The effects of decalcification rate include the concentration of acid and alkali, the temperature of decalcification and the time of processing significantly. Huang Yu et al. [6] adopted hydrochloric acid as the decalcification solution, and took the scales of tilapia as the raw materials. Decalcification rate was $99.55 \%$, the results show that: organic impurities and metal elements could be removed by hydrochloric acid from the fish scale and the dissolution rate of hydroxyproline was increased in the decalcification solution. Tu Zong-Cai et al. [7] studied on dissolution rat of hydroxyproline and the content of calcium ion as evaluation index in the decalcification solution. The results showed that high concentration of hydrochloric acid would reduce the dissolution of hydroxyproline. So it needed to maintain a certain concentration of hydrochloric acid in the decalcification solution. Peng Yuan-Huai et al. [8] used citric acid to remove calcium from the tilapia scales. The decalcification rate reached up to $96.13 \%$. EDTA was selected as chelating agent. Pati et al. [9] removed calcium from the fish scale by EDTA-decalcification. The rate of decalcification achieved to the maximum value $48 \mathrm{~h}$ later. Ilona et al. [10] compared the methods of acid-decalcification and EDTA-decalcification with other methods and found that acid-decalcification was more quick and effective, but the rate of collagen would face with the phenomenon of more huge drain. It wasted more time and acid content by acid-decalcification and EDTA-decalcification which would go against decalcification in industrial production. The microwave and ultrasonic assisted was studied which could provide more secure and efficient decalcification. Zhang Ying-Jie et al. [11] studied the process optimization of calcium removal of fish scales by the microwave assisted EDTA. It was not only increasing the processing speed of the rate of decalcification but also having little influence on the characteristics of collagen.

3.1.2 Extraction of Collagen and Gelatin from Fish Scales

The degree of hydrolysis, the extraction rate and the functional properties of collagen are considered as the response factors, when collagen is extracted with the methods of acid, alkali and enzyme from the fish scales. The collagen can be divided into acid-soluble 
collagens and pepsin-soluble collagens. The extracting program, sensory properties and thermal stability are different, but the structure is similar between ASC and PSC.

\subsubsection{Extraction of Collagen with Acid}

Acidolysis extraction can destroy the ionic bonds and Schiff bonds of collagen molecules in acidic environment which would expand and dissolve the collagen fibers. Lu Zhao et al. [12] used acetic acid extracted ASC from grass carp scale, the results showed that acetic acid $1 \mathrm{~mol} / \mathrm{L}$, extracting temperature $28{ }^{\circ} \mathrm{C}$, extracting time $25 \mathrm{~h}$, the highest extraction rate was $15.33 \%$. Zeng Shao-kui et al. [13] used citric acid to extract gelatin from the fish scale. The results showed that the optimal parameters were determined to be: extracting temperature $65{ }^{\circ} \mathrm{C}$, extracting time $3.6 \mathrm{~h}$, the removal rate of $28.4 \%$. It is necessary to select a better acid with high extraction efficiency on the economic point of view. Wang Xin-Su et al. [14] extracted ASC from grass carp scale by 3 types of acid. The extraction ratios of collagen by acetic acid, citric acid and lactic acid were $45.82 \%$, $74.34 \%, 49.31 \%$. The analysis of variance showed that the extraction with citric acid resulted in the highest extraction rate which was colorless and tasteless, whereas citric acid and lactic acid tasted heavy. The extracting with citric acid was the best choice.

The technique relies on acid hydrolysis to prepare the composite collagen from discarded fish scale, but the tryptophan and other amino acids will be destroyed. The pretreatment can not be used by acid decalcification too, or the residue of other proteins will be left. The crude protein content will decrease in the finished product. By using acidolysis extraction to produce the collagen has to be purified and serious corrosion of equipment occures by acid. It is not suitable for industrial production, so the method of optimization should be considered with acid enzymatic, ultrasonic-assisted and microwave-assisted. Hu Jian-Ping [15] decalcified by acetic acid, hydrochloric acid and citric acid, and extracted the collagen by pepsin. The decalcification rate was acetic acid > citric acid > hydrochloric acid, where the extraction rate was $73.32 \%$. Yuan Yuan et al. [16] extracted collagen from fish scales by the process of acetic acid with microwave-assisted process, the results showed that the best extraction conditions were determined as below: power $400 \mathrm{~W}$, extraction time 5 min, the extraction rate was $41.37 \%$. The extraction time was shorter. The extraction rate was higher than the acetic acid alone.

\subsubsection{Extraction of Collagen with Alkali}

There are a lot of basic groups and acidic groups in the collagen. Based on the acid-base neutralization under the condition of alkaline, cleavage occurring in peptide-chain of collagen is cleavage. The collagen is dissolved and degraded in the alkali dissolution. The alkaline method quickly and thoroughly uses bone collagen with higher crosslinking degree, but the production cycle is longer, the productivity is lower, the protein is easily denatured, the phenomenon of racemization will be appeared, peptide chains easily hydrolyze, isoelectric point is reduced, so the extraction of collagen is less with alkali. The extractant includes $\mathrm{CaO}, \mathrm{Ca}(\mathrm{OH})_{2}, \mathrm{Na}_{2} \mathrm{CO}_{3}$ etc. The effects are analysed on the extraction yield of collagen, including concentrations of alkali, the time of processing, alkali processing periods. Gu Yang-Juan [17] compared with the extracting modes of alkali, acid, enzymatic which made scales gelation. The extraction rate of collagen was lower by alkali, and freezing capacity was smaller, it was not recommended to use by alkali.

\subsubsection{Extraction of Collagen with Enzyme}

The collagen is isolated by enzyme hydrolysis extraction from fish scale. The three-screws structure only unfolds without changing in the collagen which will improve the solubility. The reaction condition is mild, higher extraction rate, shorter time and no racemization effect by enzymatic extraction. The enzymes used were papain, alkaline protease, pepsin, etc. For factors, such as enzyme concentration, the 
ratio of material to liquid, solid-liquid ratio and temperature and hydrolysis time were investigated. The process of extraction of collagen by the aided process was studied too. The processes of enzyme were divided into single enzyme hydrolysis and multi-enzyme hydrolysis. Wang Chun et al. [18] used to extract collagen from tilapia scale with papain. The results showed the optimum condition: enzyme concentration $2.5 \%$, the temperature $50{ }^{\circ} \mathrm{C}$, hydrolysis time $5 \mathrm{~h}$, where the collagen extraction rate was obtained at the highest level with the advantage of the water capacity and foam stability. Tang Xu et al. [19] studied on the production of alkaline protease by microorganism which hydrolyzed the fish scale. The parameters were as follows: enzyme treatment temperature $60{ }^{\circ} \mathrm{C}$, enzymolysis time $1.5 \mathrm{~h}$, concentration of enzyme $3 \%$, and enzyme substrate ratio $10 \%$. Under these conditions, the extraction yield was $32.38 \%$, molecular weight was distributed between 1 6 ku. Molecular weight was smaller which could be used in cosmetics. Toshiyuki Ikoma et al. [20] removed calcium by EDTA method, pepsin hydrolyzed fish scales, and extracted more than 33.6\% collagen which contained aminoacetic acid. In order to obtain the highest extraction yield, the domestic scholars compared with the titer of enzymatic hydrolysis in several kinds of enzyme and chose the best enzyme. Hu Jian-Ping et al. [21] used variety of enzymes to hydrolyse silver carp fish scales. The results showed the rate of collagen from silver carp scale were: pepsin $>$ papain $>$ trypsin.

The fish scale can be hydrolysed step by step by two or more than two kinds of enzyme or by a compound enzyme. The molecular of mixed amino acids and peptides was relatively smaller and higher active in the enzymatic hydrolysate. The single enzyme or compound enzyme can increase extraction rate. But the bitter peptides have the same range of molecular weight as the above. Cheng Lu [22] studied on the experiments of two-step-hydrolysis method. The optimal factor level combination can be determined: pepsin, papain and neutral protease. The degree of hydrolysis was $55.74 \%$, the content of peptides was $164.67 \mathrm{mg} / \mathrm{g}$. Wang Yan-Rong et al. [23] extracted collagen by the compound enzymes ( $m$ Alcalase:m PTN = 1:2). The hydrolysis of collagen reached the highest degree. The result showed that the best conditions of composite enzymatic hydrolysis were: the enzymatic hydrolysis yield was $13.24 \%$, the protein recovery of derivative was $96.37 \%$, the degree of hydrolysis peptide yield achieved $83.13 \%$. It was more effective to improve the extraction rate of collagen with other auxiliary methods of microwave or ultrasonic. Hu Jian-Ping [24] used the ultrasound combined with pepsin which extracted collagen, the ultrasonic time $230 \mathrm{~min}$, enzyme amount $350 \mathrm{mg} / \mathrm{g}$, solid-liquid ratio $1: 45 \mathrm{~g} / \mathrm{mL}$, the extraction yield $28.53 \%$. Using the ultrasonic, microwave, ultramicron comminution technology, Hu Fang-Yuang [25] extracted collagen from bighead carp scale by alcalase. The best conditions of composite enzymatic hydrolysis were ultramicron comminution. The degree of hydrolysis was $24.22 \%$, the recovery of protein was $95.76 \%$.

\subsection{Extraction of Hydroxyapatite from Fish Scale}

The hydroxyapatite (HAP) and calcium phosphate is enriched in the inorganic substance of fish scales. The HAP has good biocompatibility, activity and high compatibility. The HAP can be used to repair and replace biological hard tissues. It also can absorb heavy metals and be applied to waste water treatment.

\subsubsection{Preparation of Hydroxyapatite}

It is seen from few reports that HAP is prepared from the fish scales. HAP is mainly composed of high temperature calcining, alkali dissolution method and synthesis of acid soluble with alkali. Submicron hydroxyapatite is prepared by calcination at high temperature from the fish scale which has been removed collagen. Nanometer hydroxyapatite is prepared from the fish scale which has been removed collagen by lye, then washed and dried. When the 
hydroxyapatite is prepared by calcination at high temperature, the temperature must be strictly controlled. The HAP is easily transformed to hypoxia HAP at higher temperature. Hypoxia HAP still keeps the crystal structure of HAP, but it will reduce biological activity. Sewa et al. [26] prepared the HAP with the cleaning fish scales in a $\mathrm{KOH}$ solution at moderate temperature, then dried. The optimum conditions were as follows: calcined temperature $900{ }^{\circ} \mathrm{C}$, calcined time $1 \mathrm{~h}$. The ratio of calcium to phosphorus is different when different kinds of scales are calcined at high temperature. Yi-Cheng Huang et al. [27] prepared hydroxyapatite by calcination under temperature $800{ }^{\circ} \mathrm{C}$ for $4 \mathrm{~h}$ after the scale has been hydrolysed by the protease and flavor enzyme in turn. The ratio of calcium to phosphorus was 1.76, hydroxyapatite had good porosity and the surface roughness increased too.

\subsection{Extraction of Lecithin from Fish Scales}

Lecithin is a phospholipid mixture which is freely soluble in acetone solution, but unsolvable in ethanol solvents. Lecithin can effectively improve the function of nerve, prevent cardiovascular disease and have function of anti-aging. The optimum extraction technology was as follows: at first, lecithin is extracted with acetone from fish scales, then we dissolved the filter cake of acetone by ethanol and then the remainder filtrate is recovered to prepare lecithin. The lecithin is precipitated by a large amount of acetone and the crude lecithin was obtained. Wu Ji-kui et al. [28] obtained the highest lecithin rate, abstraction temperature $25^{\circ} \mathrm{C} ; 1 \mathrm{~h}$ (abstraction time); weight of fish:scale-volume of ethanol = 1:20 (mL/g); 85\% ethano1. Gao Meng-Xiang et al. [29] used microwave-assisted method to extract lecithin from the fish scales of silver carp. The optimum conditions that lecithin was abstracted with ethanol were: $95 \%$ ethanol volume of $50 \mathrm{~mL} / \mathrm{g}$, extracting time $4 \mathrm{~min}$, power $240 \mathrm{w}$, lecithin extraction amount is 23.87 $\mathrm{mg} / \mathrm{g}$. Abroad scholars adopted a new extraction method which stab-broken the cells of cyprinoid scale at a speed of 20,000 vibrations per second by titanium-krypton needle in Research Center of Santiago American. It interrupted the molecular structure of phospholipids.

\subsection{Extraction of Guanine from Fish Scales}

Guanine is found in the cells protoplast of fish scale and can synthesize 6-TG which has the significant anticancer property. At first the scales were put into the reactionary pot of acid proof, then $\mathrm{HCl}$ was added, surfactant $\mathrm{T}$ with heating and hydrolyzing. Standing crystallization was applied after filtration, we can obtain pure guanine on the conditions of decoloration. It will affect the extraction yield of guanine, including concentration of hydrochloric acid, the quantity of the surfactant, extraction temperature, extraction time. Zeng Xiao-Dan [30] extracted guanine from grass carp fish scales. The optimum conditions were as follows: hydrochloric acid $5.0 \mathrm{~mol} / \mathrm{L}$, extraction temperature $98{ }^{\circ} \mathrm{C}$, extraction time $2 \mathrm{~h}$, the extraction ratio reached $0.39 \%$.

\subsection{Extraction of Scleroprotein from Fish Scales}

After the collagen is extracted from the scales, the fish scale contains a amount of scleroprotein which is insoluble in water, salinity, dilute acid or dilute alkali, scleroprotein is an important component of the connective tissue in animals. The scleroprotein lacks some individual essential amino acids, the nutritive value is relatively low, and the molecular weight is large, it is also difficult to be absorbed by the animal directly. The scleroprotein is obtained after the scale is treated by $\mathrm{H}_{2} \mathrm{~S}$ or NaHS, then acid treatment process. The species and the degree of comminution are the most significant parameter of extraction rate in the fish scale. The scleroprotein can be hydrolyzed into small molecular peptides by trypsin and papain which have stronger biological activity. The small molecular peptides can be easily absorbed. Then a new method is found that production of new protein feed contains 
rich small peptides with vacuum spray drying. Chen Li-Juan et al. [31] hydrolyzed the scleroprotein of cyprinoid scales, then supplemented with tryptophan, a protein with good nutritional value was found.

\subsection{Other Extractions from Fish Scales}

There is stronger reducing property and anti-lipid peroxidation in Glycoconjugate which is mainly extraction from marine animal, but there is less research on the freshwater fish. Cao Dong-Xu et al. [32] extracted Glycoconjugate from the carp fish scales by alkali. The rational conditions were: 0.3 $\mathrm{mol} / \mathrm{L} \mathrm{NaOH}$ solution, and the ratio of solid-liquid 1:5, extraction temperature $25{ }^{\circ} \mathrm{C}$, extraction time $18 \mathrm{~h}$. The result showed that the highest extraction rate was obtained. The fish scales could continue to be decalcified and extracted collagen after the Glycoconjugate has been extracted. The study provided the reference basis for the development and application from the fish scales. There is a material with metallic luster and silver white colour on the surface of fish scales, which can be used in biochemical preparation and paint industry. The fish scale can continue to process into fish meal, and at the end it can realize maximum economic benefit.

\section{Comprehensive Extraction from Fish Scales}

It will produce amounts of by-products with nutrients and functional components when effective ingredients are extracted from the scale. For example, it will produce about $40 \%$ residue after collagen is extracted from fish scales, the residue content can be made into hydroxyapatite or soluble scleroprotein; it will produce a large deal of waste liquid that contains calcium hydrogen phosphate after decalcification, the waste liquid can be made into feed additives. Wang Nan-Ping [33] extracted calcium hydrogen phosphate with $\mathrm{NaOH}$ which had been added in the waste acid after decalcification. Its optimum conditions were: the content of calcium phosphate was $14.98 \%$, the calcium content was $22.62 \%$, the ratio of calcium to phosphorus was $1.51 \%$. The comprehensive utilization of by-products can effectively save resources, protect the environment and improve the economic value of fish scales.

The scholars explored comprehensive extraction as a new research direction in this field. Zeng Fang [34] studied the production technology of gelatin and hydroxyapatite from the fish scale. It is an important step to study on comprehensive extraction of fish scale. Wang Xi-Bo [35] established co-production process of organic calcium, collagen and collagen polypeptide from fish scale, designed the production process and the critical control points which provided the theoretical basis for the industrialized production of fish.

\section{Application Prospects of the Fish Scale}

The effective ingredients from the scale have high value. It is widely used in the food industry, cosmetics, biomedical materials field. The extraction technology of scale has great development, but the extraction process needs to be improved in many aspects. For example, the steps of acid extraction are too tedious and extract has weak acidity which is disadvantage for the medicine, food, cosmetics and other industries. Enzymatic extraction is simple, but the hydrolysate contains bitter peptides and the bitter peptides need be removed in the subsequent processing. So it is unfavorable for the food industries. The acidic conditions of enzymatic hydrolysis may affect collagen characteristics. The molecular weight of collagen under $2 \mathrm{ku}$ can be absorbed by the skin, but collagen rarely reaches the level of small molecular weight, it will affect the useful of collagen in the cosmetics industry. A new and more efficient extraction method on the basis of previous studies may be explored. Study on the extraction of ingredients which have been applied to industrial production, has the high economic significance which will improve marine added value, and promote the 
industrial production of collagen and hydroxyl from the fish scale. But the fish enrich heavy metal. We should pay more attention to fish habitats and growth environment factors when we extract ingredients. The extraction technology of effective ingredients can be used as a theoretical basis for the development and utilization of fish scales, and it also provides technical support for the sustainable development of fishery.

\section{References}

[1] Duan, R., Zhou, Y., L., and Zhang, Y. 2004. "Monitor the Process of the Decalcification from Fish Scales.” Journal of Huaihai Institute of Techology (Natural Science) 13 (2): 54-6.

[2] He, L. 2007. "Preparation and Development of Collagen from Fish Scale.” Fisheries Science \& Technology Information 34 (5): 210-2.

[3] Gu, Y. J., Li, J., and Li, F. W. 2012. "Research Progress of Effective Ingredients of Fish Scale" Science and Technology of Food Industry 33 (10): 415-9.

[4] Sawa, H., and Takenaka, A. 2001. "Kaoru Aoki.” Phosphorus Letter (1): 41.

[5] Ikoma, T., Kobayashi, H., and Tanaka, J. 2003. "Microstructure, Mechanical, and Biomimetic Properties of Fish Scales from Pafrus Major.” Journal of Structural Biology (142): 327-33.

[6] Huang, Y., and Wang, Y. 2012. "Optimization of Decalcification Technique of Fish Scale by Response Surface Methodology.” Journal of Fujian Fisheries 34 (2): 127-33.

[7] Tu, Z. C., Sha, X. M., and Wang, H. 2012. "Optimization of Decalcification Conditions of Bighead Carp Scale.” Food and Fermentation Industry 38 (2): 106-10.

[8] Peng, Y. H., Liu, X. M., and Li, X. Q. 2012. “Analysis on Decalcification of Fish Scale with Citric Acid.” Food Engineering 37: 132-5.

[9] Pati, F., Adhikari, B., and Dhara, S. 2010. "Isolation and Characterization of Fish Scale Collagen of Higher Thermal Stability.” Bioresource Technology 101 (10): 3737-42.

[10] Ilona, K., Elzbieta, S., and Maria, S. 2008. "Effect of Extracting Time and Temperature on Yield of Gelatin from Different Fish Offal.” Food Chemistry 107 (2): 700-6.

[11] Zhang, Y. J., Ceng, Q. X., and Ye, F. L. 2007. "Application of Decalcification with EDTA and Microwave-Assisted from Scale.” Food Industry 29 (01): 42-4.

[12] Lu, Z., Li, L. X., and Zhang, Y. F. 2013. "Study on Extraction Process Parameters of Acid Soluble Collagen from Grass Carp Scales.” Food Science and Technology 38 (5): 250-4.

[13] Zeng, S. K., Liu, K., and Wu, Y. T. 2013. "Extraction Optimization and Physicochemical Properties of Gelatin from Demineralized Scale of Oreochromis Nilotica." South China Fisheries Science 9 (2): 38-44.

[14] Wang, X. S., and Wang, Z. H. 2006. "Extraction and Application of Collagen from Fish Scale.” Modern Food Science and Technology 22 (4): 148-50.

[15] Hu, J. P. 2012. "Acid Extraction and Properties of Collagen from Fish Scales.” Grain Science and Technology and Economy 37 (3): 56-60.

[16] Yuan Y., and Hu J. P. 2013. "Study on Extraction Collagen with Microwave-Assisted from Fish Scale.” Hubei Agricultural Science 52 (3): 662-4.

[17] Gu, Y. J. 2013. "Processing Optimization and Characterization of Jelly from Grass Carp Fish Scales.” M.Sc. thesis, Shanghai Ocean University.

[18] Wang, C., Zhou L., and Zhou T. 2013. "Study on the Extraction of Collagen Protein from Tilapia Scale with Papain.” Journal of Anhui Agricultural Sciences 41 (3): 129l-2.

[19] Tang, X., Yang, L. H., Wu, Q. Q., and Xu, C. A. 2012. "Process Improvement of Hydrolysis Marine Fish Scales with Alkaline Protease.” Journal of Xiamen University (Natural Science) 51 (6): 1097-101.

[20] Ikoma, T., KobayaShi, H., and Tanaka, J. 2003. "Physical Properties of Type I Collagen Extracted from Fish Scales of Pagrus Major and Oreochromis Niloticas.” International Journal of Biological Macromolecules 32: 199-204.

[21] Hu, J. P., Wu, Q., and Chen, H. 2010. "Study on Extraction and Properties of Collagen from Silver Carp Scale.” Food Science and Technology 35 (1): 230-8.

[22] Chen, L. 2013. "Study on Preparation and Analysis of Collagen Peptide from Fish Scale.” M.Sc. thesis, Inner Mongolia Agricultural University.

[23] Wang, Y. R., Cui, C., and Zhao, M. M. 2011. "Enzymatic Hydrolysis and Ultrafiltration Separation of Collagens from Tilapia Skin and Scale.” Food and Fermentation Industry 37 (9): 133-6.

[24] Hu, J. P. 2013. "The Ultrasonic Extraction Fish Scale Collagen and in Vitro Antioxidant Effect of Research.” Food Industry 34 (2): 57-60.

[25] Hu, F. Y. 2012. "Study on Enzymatic Preparation of Oligopeptides and Analysis Antioxidant Activity from Aristichthys Nobilis Scale.” M.Sc. thesis, Jiangnan University.

[26] Sawa, H. 1996. "Separation of Hydroxyapatite from Scales of Fish.” Zairyo/Journal of the Society of Materials Science 45 (4): 455-8.

[27] Huang, Y. C., Hsiao, P. C., and Chai, H. J. 2011. "Hydroxyapatite Extracted from Fish Scale: Effects on 
MG63 Osteoblast-Like Cells.” Ceramics International 37 (6): 1825-31.

[28] Wu, J. K., and Zhang, J. L. 2005. "The Optimum Conditions for Abstraction of Lecithin from the Fish-Scale of Grass Carp.” Journal of Shanghai Fisheries University 14 (4): 428-31.

[29] Gao, M. X., and Liu, T. L. 2006. "Microwave-Assist Extraction Technology of Lecithin from the Fish Scale.” Food and Machinery 22 (4): 16-8.

[30] Zeng, X. D. 2012. "Study on Technology and Quality of Extraction Guanin from Grass Carp Scale.” Modern Agricultural Science Technology 1: 15-6.

[31] Chen, L. J., Wu, W. H., and Wang, N. P. 2011. "Evaluation of Scleroprotein Nutritional in the Carp Scale.” Feed Research 34 (9): 63-6.
[32] Cao, D. X., Yuan, B., and Liu, A. J. 2007. "Research on the Isolation of a Glycoconjugate from Fish Scale and Its Antioxidant Activity.” Food Research and Development 28 (11): 151-4.

[33] Wang, N. P., He, L., Cao, J., and Guo, X. Y. 2014. "Review of Comprehensive Equipment and Technology of Fish Scale.” Fishery Modernization 41 (1): 46-52.

[34] Zeng, F. 2013. Study on Comprehensive Extraction of Fish Scale Collagen, Gelatin, Hydroxyapatite. Nanchang: Nanchang University.

[35] Wang, X. B. 2013. “Co-production Process of Organic Calcium, Collagen and Collagen Polypeptide from Fish Scale and Plant Design.” M.Sc. thesis, College of Food Science and Technology Hua Zhong Agricultural University. 